\title{
Understanding the decomposition reaction mechanism of chrysanthemic acid: a computational study
}

Shabaan AK Elroby ${ }^{1,2^{*}}$ and Saadullah G Aziz ${ }^{1}$

\begin{abstract}
Background: Chrysanthemic acid (CHA) is a major product from the photodecomposition of pyrethrin which is an important class of pesticide compounds.

In the following paper, Hybrid density functional theory (DFT) calculations of the potential energy surface (PES) for three possible channels decomposition of chrysanthemic acid (cis-trans isomerization, rearrangement and fragmentation) have been carried at the B3LYP/6-311+G** level of theory. DFT was employed to optimize the geometry parameters of the reactants, transition states, intermediates and products based on detailed potential energy surfaces (PES).

Results: Our results suggest that all three pathways of CHA are endothermic. DFT calculations revealed that the activation barriers for cis-trans isomerization are low, leading to a thermodynamically favorable process than other two pathways. We also investigated the solvent effect on the PES using the polarizable continuum model (PCM). In addition, time-dependent density functional theory (TDDFT) calculations showed that these reactions occur in the ground state rather than in an excited state.

Conclusion: The rearrangement process seems to be more favorable than the decomposition of CHA to carbene formation. The solvent effect calculations indicated no changes in the shape of the PES with three continua (water, ethanol and cyclohexane), although the solvents tend to stabilize all of the species.
\end{abstract}

\section{Background}

Pesticides are essential products in everyday life. The natural pyrethrin insecticides have the desirable environmental properties of being both non-toxic to mammals and non-persistent $[1,2]$. Experimental studies have been reported that pyrethrins degrade rapidly when exposed to sunlight [3]. Chrysanthemic acid [2, 2-dimethyl-3-(2methyl-1-propenyl)] is one of the products from pyrethrin photodecomposition and is widely used as the acidic part of synthetic pyrethroidin insecticides [4-9]. The photochemical degradation of the acid components of pyrethrins has been examined in numerous studies. Sasaki et al. [10] and Ueda and Matsui [11] found that carbons 1 and 2 (Scheme 1) of the main cyclopropane group are cleaved, subsequently leading to the formation

\footnotetext{
* Correspondence: skamel@kau.edu.sa

${ }^{1}$ Chemistry Department, Faculty of Science, King Abdulaziz University, Jeddah, Saudi Arabia

Full list of author information is available at the end of the article
}

of a diradical according to Elliot and Janes [12]. The photochemistry of simple cyclopropanes has been examined by several workers [13-18] and the fundamental photochemical transformations are generally observed. For instance, the photodegradation of pyrethrins in sunlight is rapid and results in the isomerization of the side-chains, photooxidation to a variety of carboxylic acids, and isomerization of the cyclopropane acids.

The main objective of this project was to carry out a theoretical study to determine the reaction mechanism of the decomposition of chrysanthemic acid. This investigation may improve our knowledge about the potential energy surface (PES) of these important compounds. We explored the potential energy surfaces of the ground state and the lowest singlet and triplet excited states and the interplay between them. DFT calculations have been used successfully in calculating transition structures [19] and the reaction parameters of various reactions, such as pericyclic rearrangements, cycloadditions 


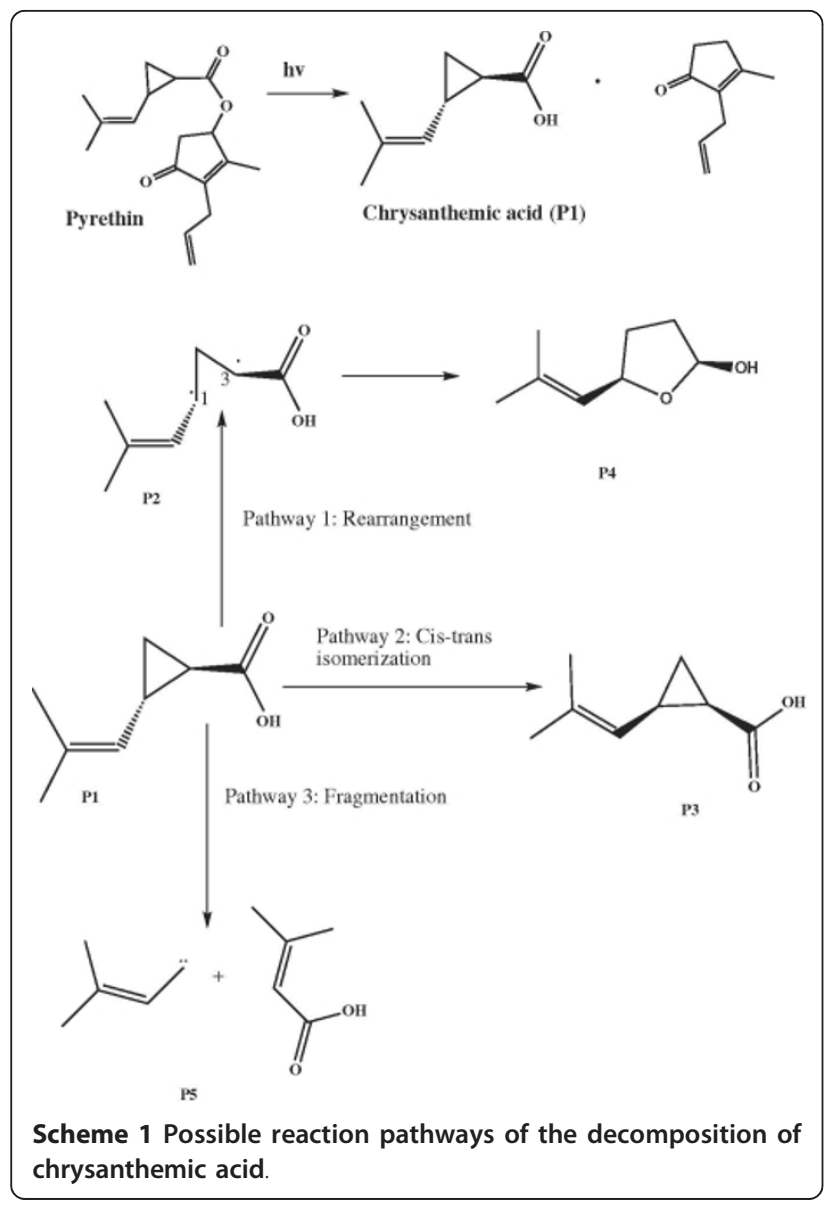

[20] and bimolecular $\mathrm{SN}^{2}$ reactions [21]. In the present work, we use the density functional (DFT) approach to undertake a theoretical investigation of the mechanism of photochemical decomposition of CHA. More specifically, we calculate structures and relative energies of reactant, products, intermediate and transition states involved in all the three suggested pathways (cis-trans isomerization, rearrangement, fragmentation). For a more complete study we also carried out the effect of solvent on the PES using the polarizable continuum model (PCM).

\section{Computational Details}

All calculations were performed with the Gaussian03W [22] program, running on a Pentium IV personal computer. We used restricted and unrestricted B3LYP gradient corrected exchange-correlation functional $[23,24]$ in combination with the $6-311+G^{* * *}$ basis set [25-27]. In our computational investigation, we determined the location of the minima and transition structures of the singlet state surfaces. For equilibrium geometries and transition states, the nature of the critical points was confirmed by an analytic frequency computation. All the transition states have imaginary frequencies. We carried out IRC calculations to confirm that the transition stats connect to right minima. Zero-point vibrational energy corrections (ZPVE) were estimated at the same theory level at which optimization was carried out and $E_{\text {total }}$ was calculated as $E_{\text {opt }}$ (optimization energy at equilibrium geometry) + ZPVE. Basis set superposition error (BSSE) corrections [28] were used to obtain more reasonable total energies in fragmentation reaction (pathway 3). Subsequently, the polarizable continuum model (PCM) [29] was applied considering water, ethanol, and cyclohexane as solvents $(\varepsilon=78.3553,24.852$, and 2.0165 , respectively). Atomic charges were derived by natural [30] population analyses.

\section{Results and discussion}

Three pathways are possible for the decomposition reaction of chrysanthemic acid, as described in an introduction and as displayed in Chart 1: trans-cis isomerization (P1-P3), fragmentation, cleavage of the 1, 3 and 1, 2 bonds (P1-P5) and rearrangement (P2-P4). This project was designed to explore the PES for the photodecomposition of chrysanthemum acid, which is a major product from the decomposition of pyrethrine pesticides. To reduce the time necessary for calculations, we replaced the methyl groups in CHA with hydrogen atoms. The structure of CHA is depicted in Figure 1.

\section{Cis-trans isomerization pathway}

To investigate the cis-trans isomerization mechanism of CHA (P1-P3), the potential energy profile was generated by scanning the C12-C1-C3-C8 ( $\theta)$ angle (Figure 1) from 0 to $180^{\circ}$ in the $S_{0}$ state. The different conformations located during the cis-trans isomerization of CHA are displayed in Figure 2. The geometrical parameters

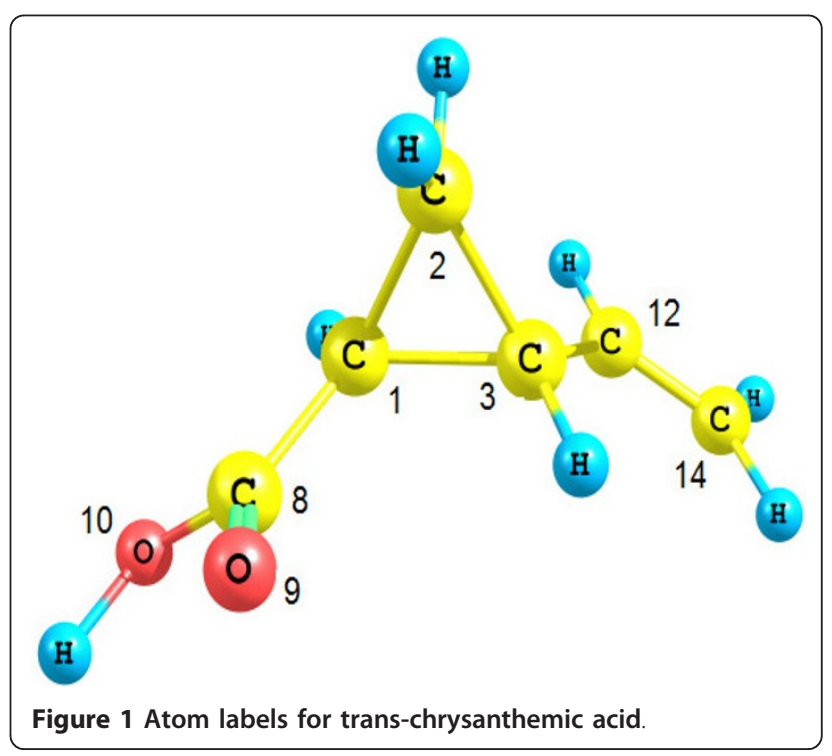




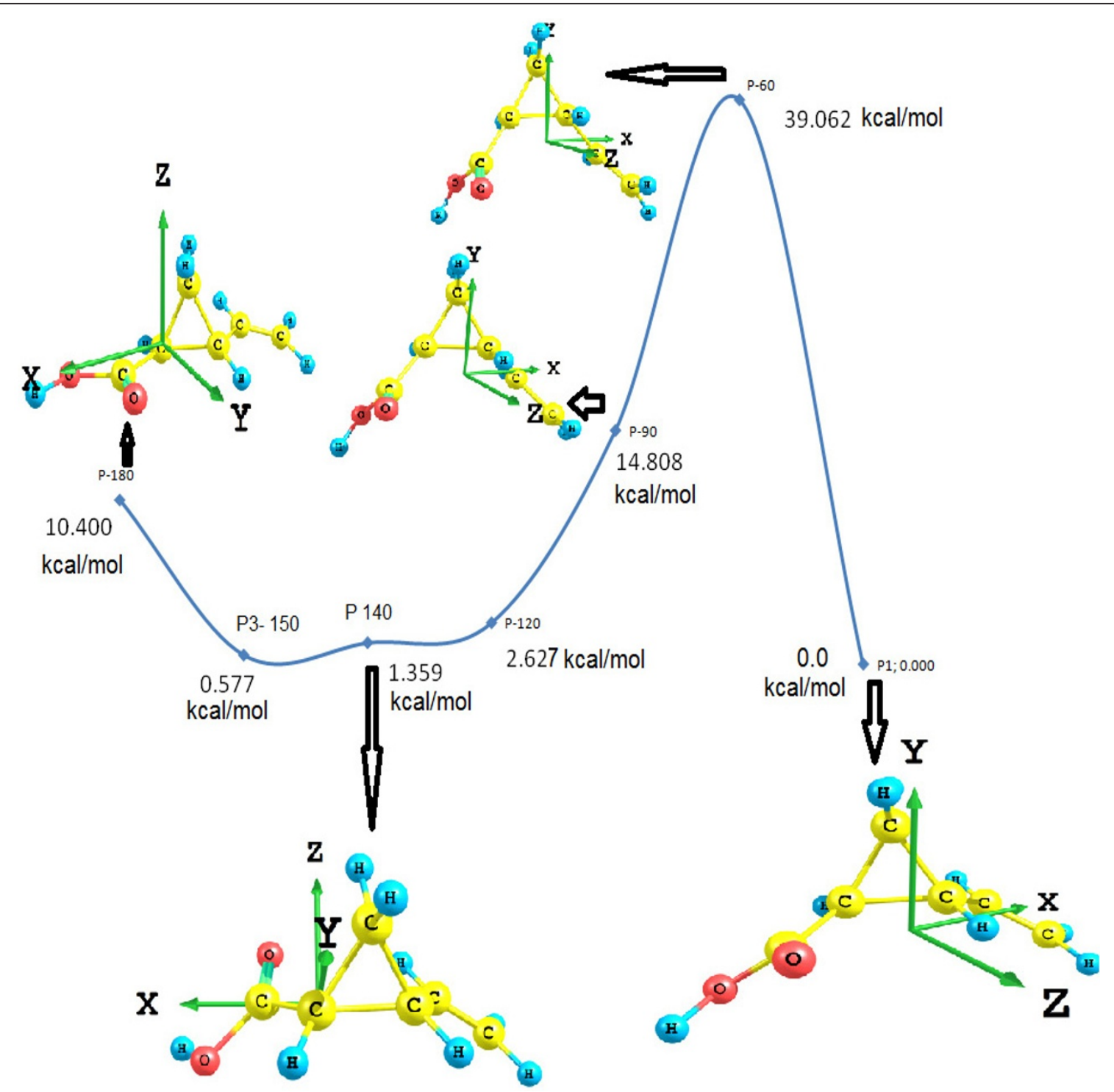

Figure 2 Potential energy profiles along the rotation around the C12-C1-C3-C8 dihedral angle and some optimized structures (angles and relative energies are given in angles and $\mathrm{kcal} / \mathrm{mol}$, respectively).

for the $\mathbf{P 1}$ and $\mathbf{P} 3$ species are similar. The values of C12-C1-C3-C8 dihedral angles are $150.0^{\circ}$ and $2.72^{\circ}$ for $\mathbf{P 1}$ and $\mathbf{P 3}$ isomers, respectively. These results show that the two branched groups of CHA are approximately in a plane in the cis form (Additional file 1). By inspection Figure 2, one trans conformer associated with the dihedral angle $\mathrm{C} 12-\mathrm{C} 1-\mathrm{C} 3-\mathrm{C} 8=180^{\circ}$ is less stable than cis form, P1 by $\sim 10 \mathrm{kcal} / \mathrm{mol}$. However, the energy difference between the two minimum isomers (P1 and P3) is only $0.577 \mathrm{kcal} / \mathrm{mol}$. The potential curve calculated at the B3LYP/6-311+G** level of theory possesses minima at $\theta=2.72^{\circ}$ and $150^{\circ}$ in the cis and trans isomers, respectively. The structure with $\theta=60^{\circ}$ is predicted to be a transition state with an imaginary frequency of i1130.91 $\mathrm{cm}^{-1}$. The energy barrier was calculated to be $39.062 \mathrm{kcal} / \mathrm{mol}$. No significant differences were found in all of the other geometrical parameters between the two isomers (cis and trans), as shown in Figure 3. The optimized geometries of the reactants, transition state and products are shown in Figure 3. A more detailed and comparative analysis of the geometric parameters for the P1-P3 reaction revealed that a drastic geometric reorganization occurs from $\mathbf{P 1}$ to the transition state (TS). The $\mathrm{C} 1-\mathrm{C} 2$ distance decreases from $1.539 \AA$ in $\mathbf{P} 1$ to $1.492 \AA$ in TS. The C3-C2 and C3-C1 distances increase from $1.512 \AA$ and $1.499 \AA$ in $\mathbf{P 1}$ to $1.531 \AA$ and $1.518 \AA$ in TS, respectively. The average total lengths of the three $\mathrm{C}-\mathrm{C}$ bonds in the ring are 

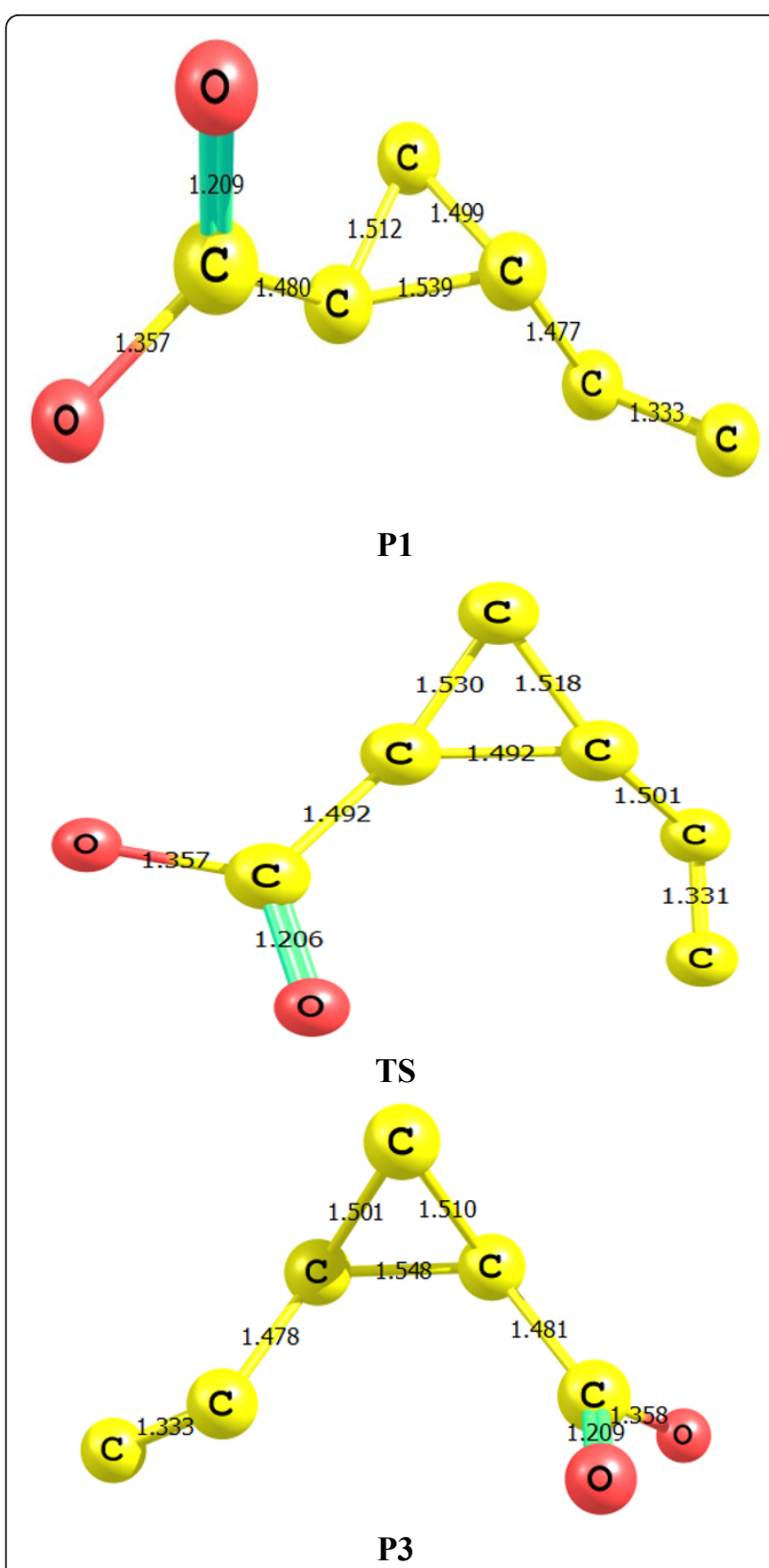

Figure 3 Geometries of the stationary points for the first suggested pathway (P1-P3); distances are given in $\AA$.

exactly the same $(1.515 \AA)$, indicating that the ring does not open during rotation about the $\mathrm{C} 12-\mathrm{C} 1-\mathrm{C} 3-\mathrm{C} 8$ dihedral angle. These results indicate that the cis and trans forms of CHA are found in similar proportions, in accordance with previous experimental observations [31].

\section{Rearrangement Pathway}

The rearrangement of CHA (P1) to produce lactone (P4) was investigated using the B3LYP/6-311+G** level of theory. Our computations predict that the pathway of the rearrangement of CHA occurs in stepwise mechanism. As shown in Figure 4, the potential energy surface of rearrangement process contains two transition states and diradical intermediate. The electronic energies, the electronic energies plus the corresponding zero-point vibrational energy (zpve) corrections, and relative energies for all the species are reported in Table 1 and take the corresponding reactant as a reference. All calculations are carried for all species in potential energy surface for rearrangement process using the restricted and unrestricted B3LYP/6-311+G** level. The relative energies in Figure 4 indicate that the rearrangement pathway for CHA is endothermic reaction where the product is at $16.8 \mathrm{kcal} / \mathrm{mol}$ above reactant. The closed-shell first transition structure on this process, TS1, is located 1.4 $\mathrm{kcal} / \mathrm{mol}$ below the second transition state TS2. According to the relative energies for the stationary points listed in Figure 5, the forward and back activation barriers are 47.5 and $32.1 \mathrm{kcal} / \mathrm{mol}$, respectively. The previous studies on the rearangment of vinylcyclopropane have reported [13] that only one transition state is located. The activation barrier in this studied had about $46.9 \mathrm{kcal} / \mathrm{mol}$ compared to $47.5 \mathrm{kcal} / \mathrm{mol}$ in our study. This result indicates that an influence of the carboxylic group on the activation energy of the rearrangement path is little. The energy difference between the final product and the intermediate species is $24.10 \mathrm{kcal} / \mathrm{mol}$ from DFT calculations. When focused on the overall energetic of the reaction P1-P4, it is evident that the reaction depends on the energetic balance due to the cleavage of a $\mathrm{C} 1-\mathrm{C} 3$ bond in the starting reactant P1and accompanied by the formation of a $\mathrm{C} 3$ - $\mathrm{O}$ bond in the rearranged carbon centered diradical intermediate. This

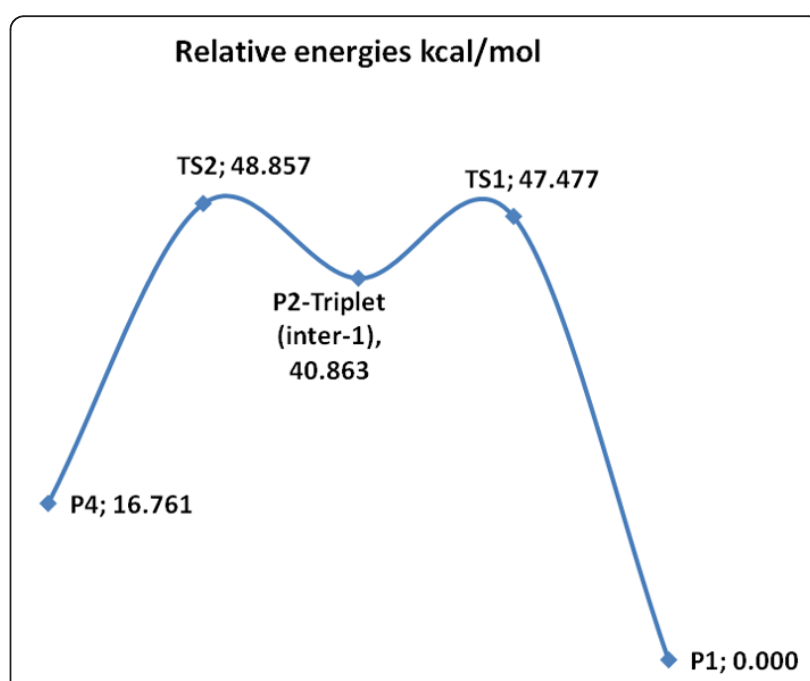

Figure 4 The calculated relative energies in $\mathrm{kcal} / \mathrm{mol}$ of the rearrangement of $\mathrm{CHA}$ at the $\mathrm{B} 3 \mathrm{LYP} / 6-311+\mathrm{G}^{* *}$ level. 
Table 1 Calculated Energies of the stationary points of rearrangement of CHA (P1-P4).

\begin{tabular}{ccccccc}
\hline Compound & $\mathbf{E}_{\text {opt/au }}$ & $\mathbf{V}_{\text {ZPE/au }}$ & $\mathbf{E}_{\text {total/au }}$ & Relative energies $\mathbf{k c a l} / \mathbf{m o l}$ & Dipole moment D.B & Imaginary frequency \\
\hline P1 & -383.98757 & 0.12936 & -383.85821 & 0.000 & 1.553 & 3.185 \\
TS1 & -383.9088 & 0.12844 & -383.78036 & 48.857 & 1.565 & -279.550 \\
P 2-Triplet (inter-1) & -383.91742 & 0.12432 & -383.79311 & 40.863 & 3.747 & 0.000 \\
TS2 & -383.90675 & 0.12419 & -383.78256 & 47.476 & 1.653 & -292.700 \\
P4 & -383.96108 & 0.12957 & -383.83151 & 16.761 & 0.000 \\
\hline
\end{tabular}
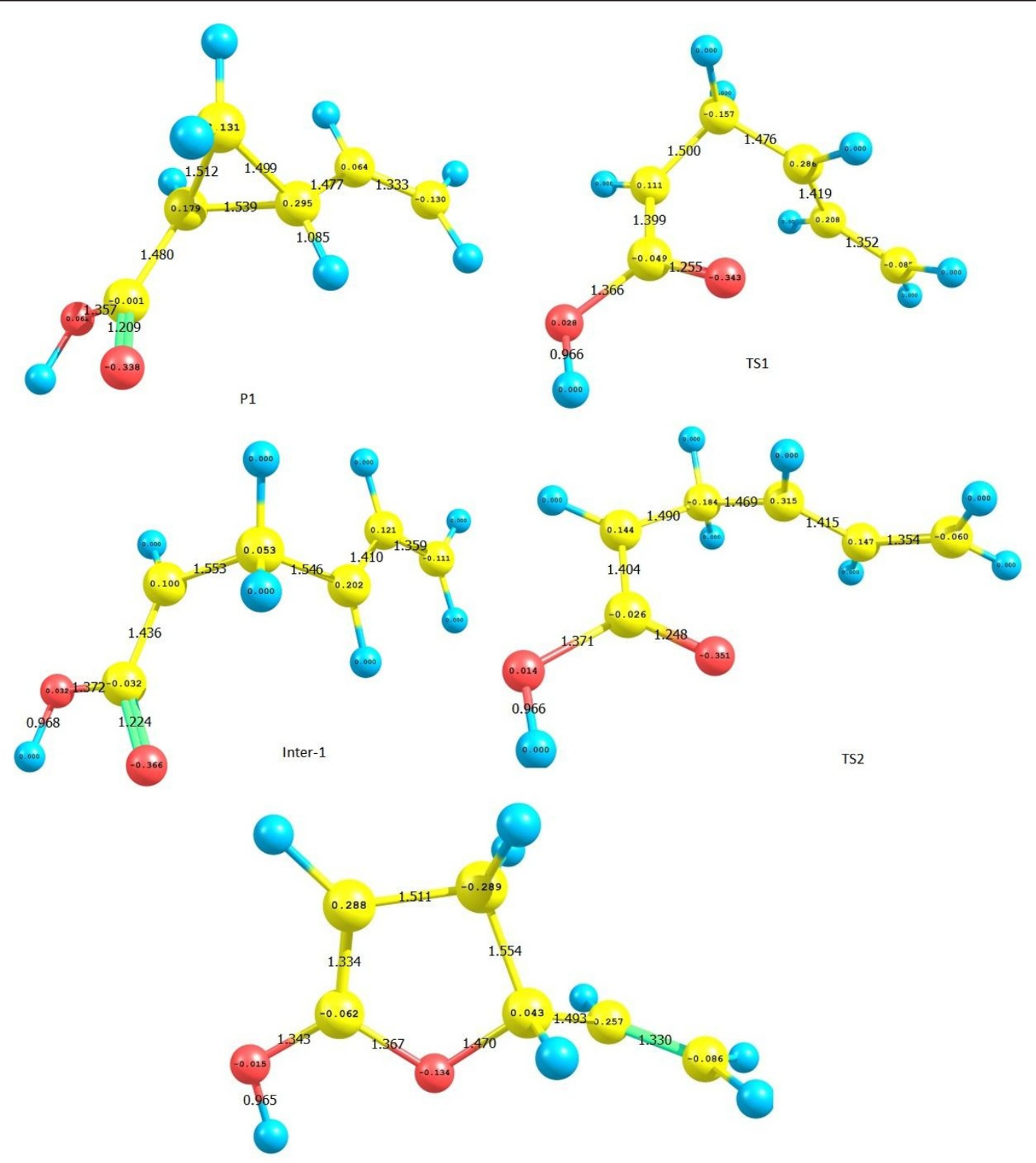

P4

Figure 5 Bond lengths and Milliken charge distribution for optimized structures of the stationary points located for the rearrangement reactions (P1-P4). Distances are given in $\AA$. 
path is started with cleavage C1-C3 bond in trans conformation P1 with decreasing others $\mathrm{C}$-C bonds except the terminal $\mathrm{CH}=\mathrm{CH} 2$. Figure 5 displays the main geometrical parameters of the equilibrium and transition structures for the formation of lactone optimized at the B3LYP level with the basis set described above. The negative charge of oxygen atom in the carbonyl group increases from TS1 (Additional file 2) with -0.343 to reach -0.351 in TS2. Also, the positive charge on $\mathrm{C} 1$ in TS1 and TS2 is 0.286 and 0.315 , respectively. These values mean that the formation bond during this pathway occurs easily between oxygen of carbonyl group and $\mathrm{C} 1$ than other carbon atoms. In conclusion, the rearrangement in the CHA followed the same route as that in the vinylcyclopropane with the slightly lowered potential barrier.

Therefore, the most important channel for CHA decomposition channels is the decomposition to carbene product via TS3, The third pathway is the fragmentation, which gives a carbene compound (P1-P5) via C1$\mathrm{C} 2$ and $\mathrm{C} 1-\mathrm{C} 3$ bond cleavage, was also investigated with the B3LYP/6-311+G** level of theory. We generated a potential energy profile by scanning the $\mathrm{C} 1-\mathrm{C} 2$ bond. Figure 6 displays the potential energy surface for cleavage of the C1-C2 bond. The total energy of the conformations along the potential surface increases with cleavage of the $\mathrm{C} 1-\mathrm{C} 2$ bond, followed by cleavage of the C1-C3 bond to produce the products, as shown in Figure 6. Inspection of the data in Figure 6 reveals an increased dihedral angle $\mathrm{C} 2-\mathrm{C} 1-\mathrm{C} 3-\mathrm{C} 12$ and decreased

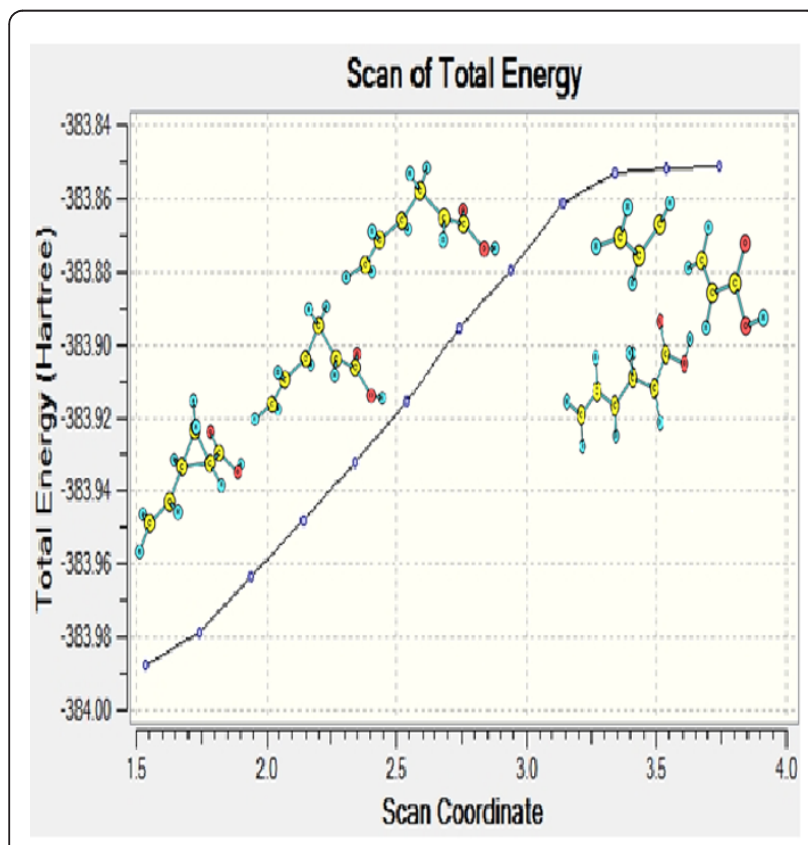

Figure 6 The potential energy surface diagram of the fragmentation process via C1-C2 cleavage (P1-P5).

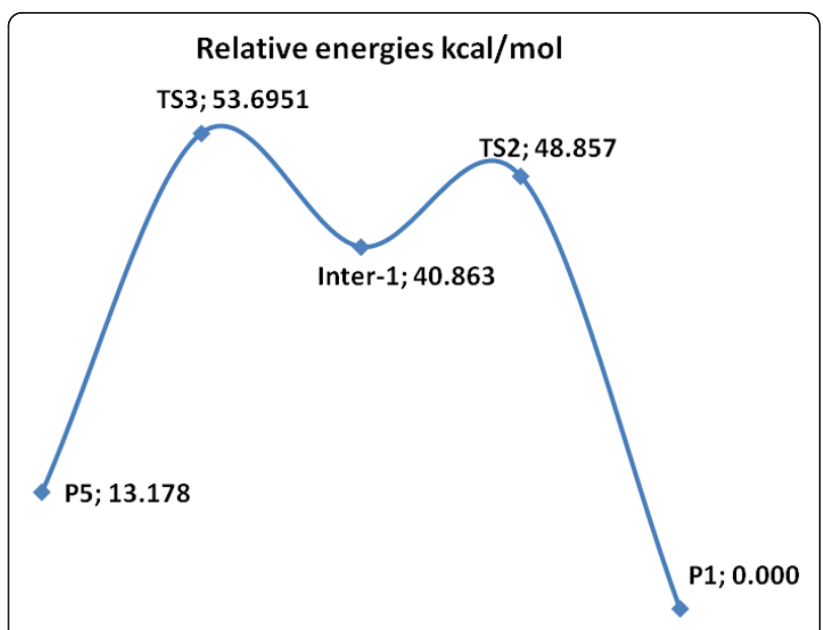

Figure 7 The relative energies for the fragmentation pathway of chrysanthemic acid.

C3-C1 bond length in TS2, indicating that the reaction appears to be concerted. The $\mathrm{C} 2-\mathrm{C} 3$ bond is the same in the two transition states. However, Figures 7 and 8 show a noticeable shortening of the $\mathrm{C} 2-\mathrm{C} 3$ bond along the fragmentation pathway. The fragmentation process occurs after two steps of successive conformational changes via two transition states, TS2 and TS3. Calculations of the harmonic vibrational frequencies (imaginary frequencies) revealed that TS2 and TS3 are true transition states and identified the atoms undergoing the main displacement in the transition structure. The relative energy values are depicted in Figure 7. These results indicate that this fragmentation is more endothermic than the other two pathways. Because it is too endothermic, this fragmentation process (P1-P5) is less favorable

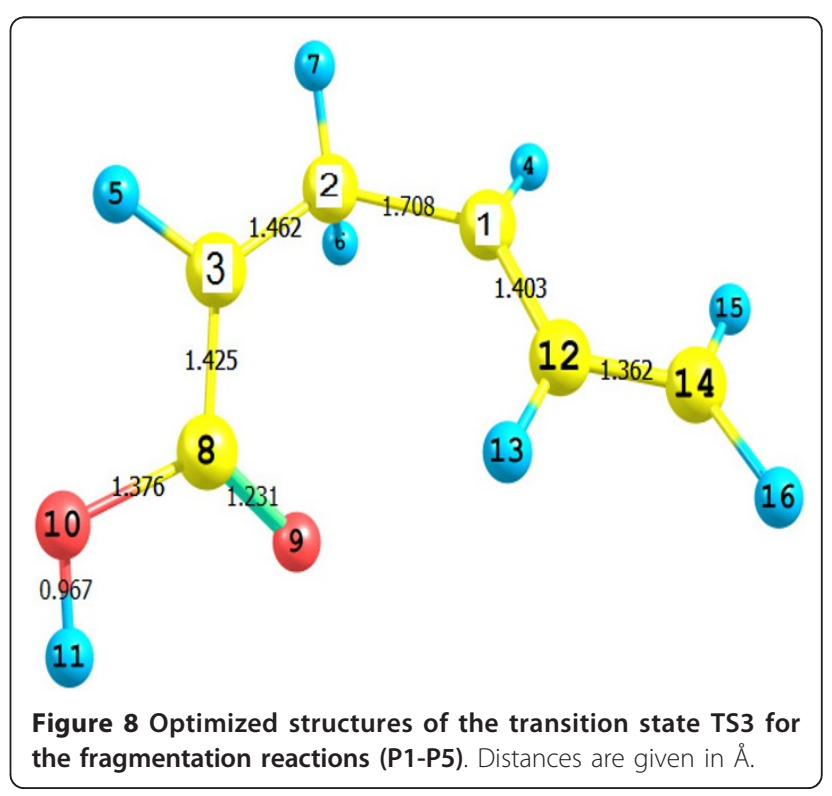


Table 2 Calculated low-energy singlet excitation energies, wavelengths, and oscillator strengths (f) for CHA (P1) using TD-B3LYP/6-311G** level.

\begin{tabular}{cccccc}
\hline Type & Oscillator strength (f) & Wave length(nm) & Excitation energy (eV) & Coefficients & Contributions \\
\hline Singlet-1 & 0.195 & 335.88 & 5.912 & 0.655 & $\mathrm{HOMO}-1->$ LUMO \\
& & & & 0.445 & $\mathrm{HOMO}->$ LUMO \\
Singlet-2 & 0.0567 & 291.7 & 5.727 & 0.549 & $\mathrm{HOMO}-1->$ LUMO \\
& & & & 0.431 & $\mathrm{HOMO}->$ LUMO \\
Singlet-3 & 0.037 & 285.91 & 6.003 & 0.658 & $\mathrm{HOMO}-1->$ LUMO \\
\hline
\end{tabular}

to proceed than the other two pathways. The endothermicity of this reaction with the BSSE correction is $71.517 \mathrm{kcal} / \mathrm{mol}$. The high endothermicity of the third pathway prevents fragmentation because it is even higher than the energy barrier for the more feasible pathway through TS3 $(47.5 \mathrm{kcal} / \mathrm{mol})$, as shown in Figure 7. This required energy is significant when compared to the more feasible pathway, as shown in Figure 4 (1.445 kcal/mol endothermic).

\section{Excitation energy}

A standard B3LYP hybrid functional was consistently employed for both geometry optimizations and TD-DFT calculations. We used the TD-DFT/6-311+ $\mathrm{G}^{* * *}$ method to determine if the reactions occur in the ground or an excited state. We calculated the first vertical excitation energies. If this energy is small, then the reaction probably occurs in the excited state. In further details about the electronic structure, we characterized the low-lying three singlet excited states within the current TD-DFT calculations as in Table 2. All excited states represent the $\mathrm{p}-\mathrm{p}^{*}$ and $\mathrm{n}-\mathrm{p}^{*}$ intramolecular charge transfer (CT) transitions. The First and second peaks originate from mixing orbitals, HOMO and HOMO-1 to LUMO. The first intense peak, dominantly describable with HOMO1 - LUMO excitation, has large f of 0.195 (Additional file 3 ). The HOMO is mainly delocalized on whole molecule whereas the HOMO-1 is located on carboxylic moiety, depicted in Figure 9. HOMO-1 - LUMO excitation therefore leads to intramolecular CT from vinyl moiety to carboxylic moiety, as illustrated in Figure 9 where the electron density increases mainly in the vinyl moiety and decreases in the carboxylic moiety. The third transition originates from HOMO-1 (mainly distributed on carboxylic moiety) to LUMO with moderate $\mathrm{f}$ of 0.037 . By inspection of the FMO correlation diagram of $\mathbf{P 1}$ as depicted in Figure 9, the HOMO is stabilized than the LUMO, which lead to the gap energy is high. The electron density is littler on C1-C3 bond in the ring this means that this bond is easy to break. Our results showed that the promotion of an electron requires a large amount of energy relative to the activation energy and the energy of the reaction. The excitation energy of $\mathbf{P 1}$ is $136.46 \mathrm{kcal} /$ mole (Table 2), which is larger than the value of barrier of the three studied pathways. These results suggest that all of the studied reactions take place in their ground state rather than in an excited state.

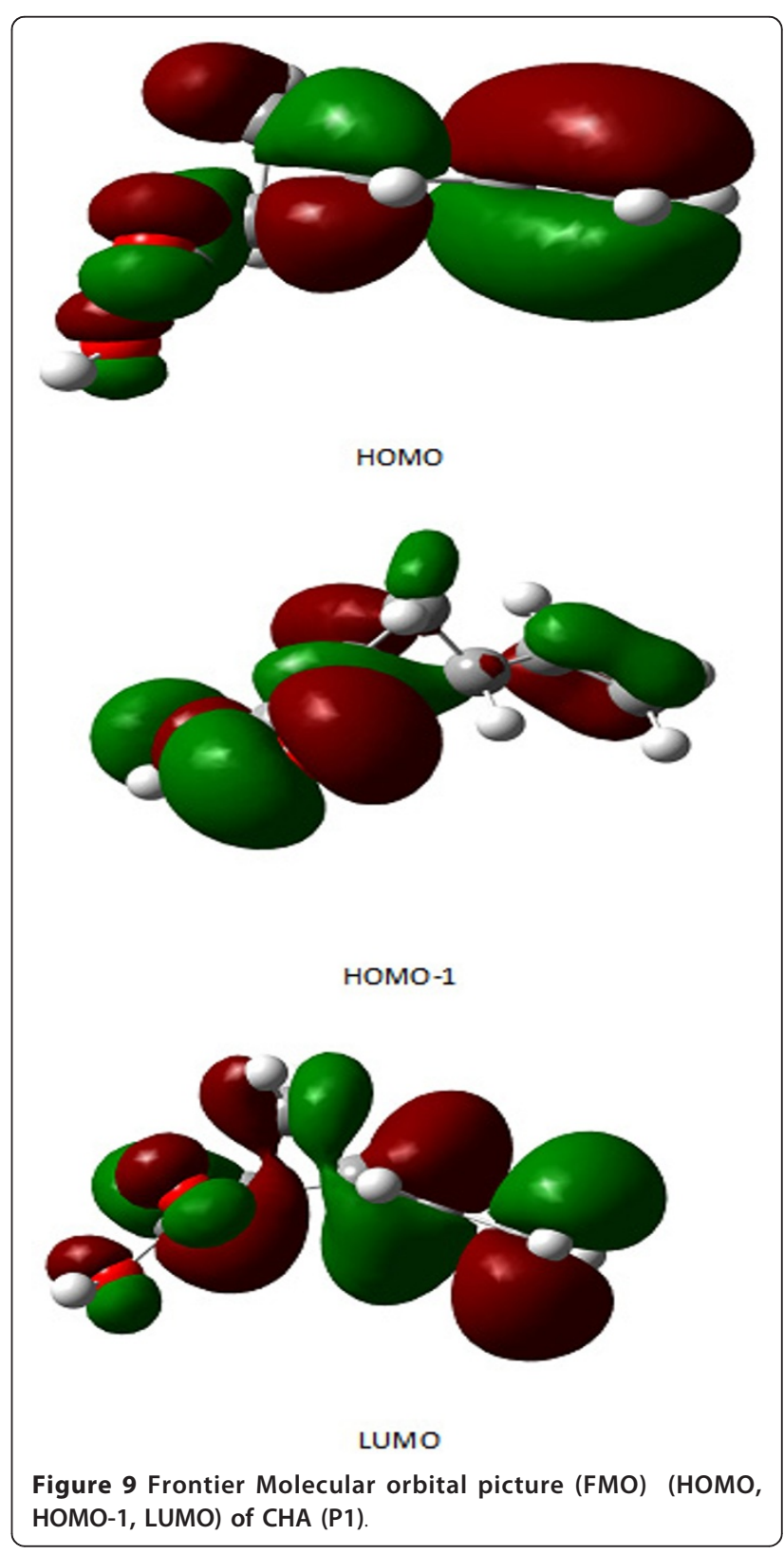




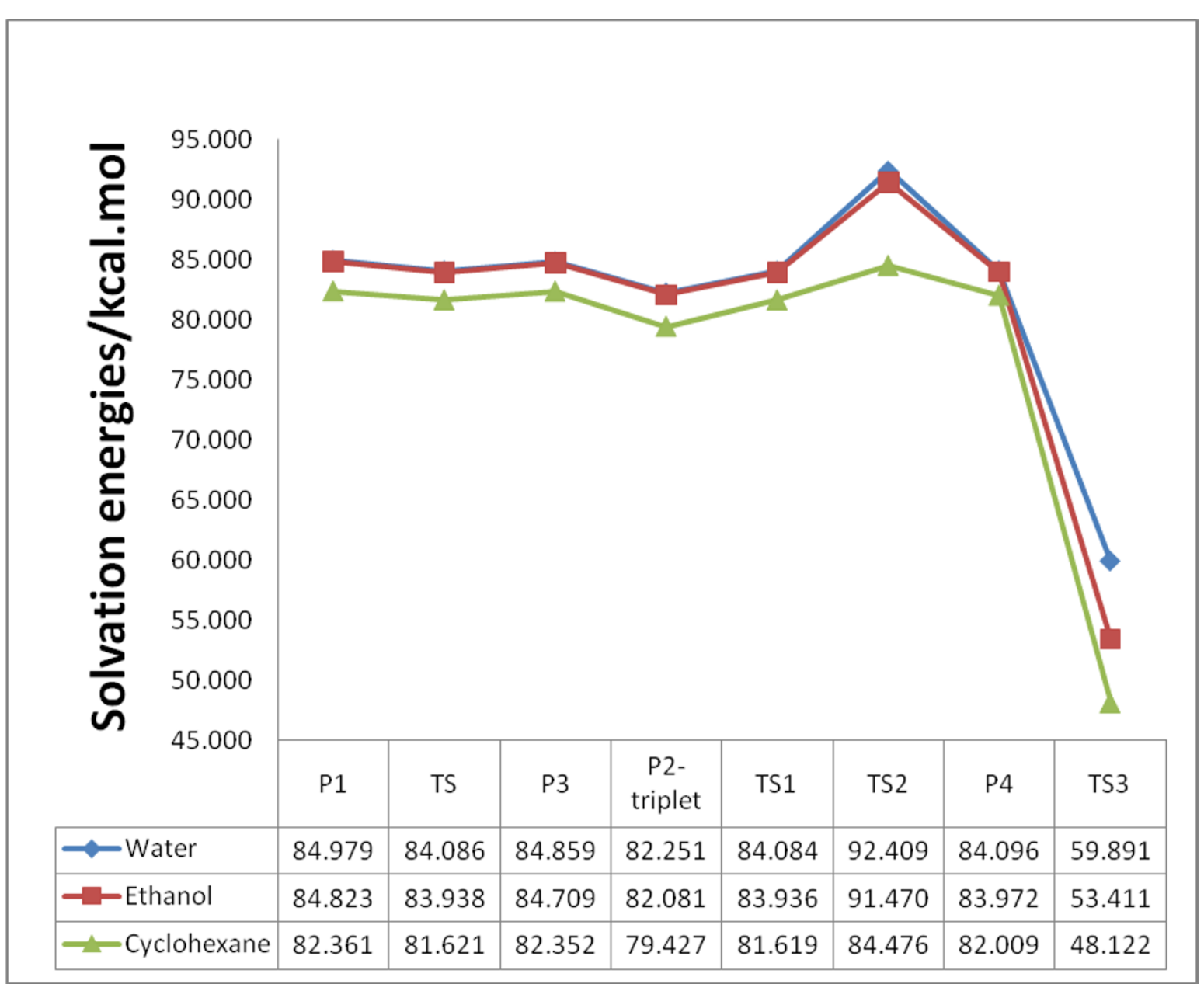

Figure 10 The solvation energies in $\mathrm{kcal} / \mathrm{mol}$ of the studied species in PES using PCM at the B3LYP/6-311+G** level.

\section{Solvent Effect}

We investigated the solvent effect using single-point SCRF calculations on optimized structures. We used an SCRF method with a polarizable continuum model (PCM) at the B3LYP/6-311+G* $\mathrm{G}^{* *}$ level (Additional file 4). The effect of different dielectric constants on the PES of the decomposition of CHA was performed using three solvents (water $=78.3553$, ethanol $=24.852$ and cyclohexane $=2.0165$ ). The solvent effect on the PES for the reaction decomposition of CHA at the DFT level of theory is schematically shown in Figure 10.

The solvent does not affect the ordering stability of the potential energy surface species. However, the solvents increase the stability of all of the reaction species with increasing dielectric constants.

\section{Conclusion}

Potential energy surface (PES) for three channels of decomposition of CHA (P1) were studied in the gas phase at the B3LYP/6-311+G* level of theory. The solvent effect on PES was also analyzed theoretically. Based on our results and analyses, we conclude the following.

1 - The calculations rationalize and verify all experimental facts. The B3LYP/6-311+G* $\mathrm{G}^{*}$ level of theory provides a reasonable way to investigate the decomposition channels of chrysanthemic acid.

2 - Cis-trans isomerization pathway is energetically favorable than the fragmentation and rearrangement pathways.

3 - The solvent effect does not affect the shape of the potential energy surfaces. In other words, the solvent effect on the reaction is small and tends to stabilize all of the isomers.

\section{Additional material}

Additional file 1: P1-Opt. Output optimized of P1 structure.

Additional file 2: NBO-TS1. Output. 
Additional file 3: P1-TDDFT. Output UV absorption calculation using TDDFT method for P1.

Additional file 4: P1-P4-inter-Cyclohexan. Output of solvent effect (single calculation) of P1-P4 intermediate. of Natural Bond Orbital calculation for TS1 structure.

\section{Acknowledgements}

This Project was funded by the Deanship of Scientific Research (DSR) King Abdulaziz University, Jeddah, under grant no. MS 11/4. The authors, therefore, acknowledge with thanks DSR support for Scientific Research.

\section{Author details}

${ }^{1}$ Chemistry Department, Faculty of Science, King Abdulaziz University, Jeddah, Saudi Arabia. ${ }^{2}$ Chemistry Department, Faculty of Science, Beni-Suief University, Beni-Suief, Egypt.

\section{Authors' contributions}

Authors initiated and designed the study. All calculations are carried out by SAE and SGA. Authors contributed to data analyses and to finalizing the manuscript. All authors have read and approved the final version.

\section{Competing interests}

The authors declare that they have no competing interests.

Received: 2 June 2011 Accepted: 30 October 2011

Published: 30 October 2011

\section{References}

1. McLaughlin GA: History of pyrethrum. In Pyrethrum; The Natural Insecticide. Edited by: Casida JE. Academic Press. New York, NY; 1973:3.

2. Todd GD, Wohlers D, Citra M: Toxicology Profile for Pyrethrins and pyrethroids. Department of Health and Human Services. Agency for Toxic Substances and Disease Registry Atlanta, GA; 2003.

3. Moorman $\mathrm{R}$, Nguyen $\mathrm{K}$ : Identification and quantitation of the six active compounds in a pyrethrin standard. J Assoc Off Anal Chem 1997, 65:921-926.

4. Yoshioka H, Miyamoto J: Synthetic Pyrethroids (1). Chem Biol 1976, 14:427-434.

5. Chang Q, Feng T, Song S, Zhou X, Wang C, Wang Z: Analysis of eight pyrethroids in water samples by liquid-liquid microextraction based on solidification of floating organic droplet combined with gas chromatography. Microchimica Acta 2010, 171:241-247.

6. Alain $K$, Willy $D$, Adrian $K$ : Novel synthesis of (d, l)-cis-chrysanthemic acid involving $a, a^{\prime}$-dibromination of 2, 2, 5, 5-tetramethylcyclohexane-1, 3dione: application to the enantioselective synthesis of (1R)-cischrysanthemic acid. Tetrahedron Letters 2009, 50:2398-2401.

7. Joly S, Jacqueline VG, UIf H: Enzymatic hydrolysis and synthesis of chrysanthemic acid esters. Enz and Microbial Tech 2005, 37:254-260.

8. Staudinger H, Ruzicka L: Insektentotende stoffe, I-VI and VIII-X. Helv Chim Acta 1924, 7:177-259.

9. Nishizawa M, Shimizu M, Ohkawa H, Kanaoka M: Stereoselective Production of (1)-trans-Chrysanthemic Acid by a Microbial Esterase: Cloning, Nucleotide Sequence, and Overexpression of the Esterase Gene of Arthrobacter globiformis in Escherichia coli. App and Env Microbiology 1995, 61:3208-3215.

10. Sasaki T, Eguchi S, Ohno M: Studies on chrysanthemic acid. 4. Photochemical behavior of chrysanthemic acid and its derivatives. $J$ Org Chem 1970, 35:790-793.

11. Ueda K, Matsui M: Studies on chrysanthemic acid. 11. Photochemical isomerization of chrysanthemic acid and its derivatives. Tetrahedron 1971, 27:2771-2774.

12. Elliott M, Janes NF: Chemistry of the natural pyrethrins. In Pyrethrum; the Natural Insecticide. Edited by: Cadisa JE. Academic Press. New York, NY; 1973:84-91.

13. Maja N, Dirk S, Olaf W, Houk KN: Computational Explorations of Vinylcyclopropane-Cyclopentene Rearrangements and Competing Diradical Stereoisomerizations. J Org Chem 2000, 65:3259-3268.
14. Neureiter NP: Pyrolysis of 1, 1-Dichloro-2-vinylcyclopropane Synthesis of 2-Chlorocyclopentadiene. J Org Chem 1959, 24:2044-2046.

15. Vogel E: Kleine Kohlenstoff-Ringe. Angew Chem 1960, 72:4-26.

16. Baldwin JE: Thermal Isomerizations of Vinylcyclo-propanes to Cyclopentenes. J Comput Chem 1998, 19:222-231.

17. Carpenter BK: Bimodal Distribution of Lifetimes for an Intermediate from a Quasiclassical Dynamics Simulation. J Am Chem Soc 1996, 118:10329-10330.

18. Klärner F-G: Walk Rearrangements in [n.1.0] Bicyclic Compounds. In Topics in Stereochemistry. Volume 15. Edited by: Eliel EL, Wilen SH, Allinger NL. John Wiley 1984:1-42.

19. Jursicin BS: Computing transition state structures with density functional theory methods. In Recent Developments and Applications of Modern Density Functional Theory. Edited by: Seminario JM. Elsevier, Amsterdam; 1996:709-741.

20. Houk KN, Gonzales J, Li Y: Pericyclic Reaction Transition States: Passions and Punctilios. Acc Chem Res 1995, 28:81-90.

21. Glukhovtsev MN, Bach RD, Pross A, Radom L: The performance of B3-LYP density functional theory in describing $S N 2$ reactions at saturated carbon. Chem Phys Lett 1996, 260:558-564

22. Frisch MJ, Trucks GW, Schlegel HB, Scuseria GE, Robb MA, Cheeseman JR, Montgomery JA Jr, Vreven T, Kudin KN, Burant JC, Millam JM, lyengar SS, Tomasi J, Barone V, Mennucci B, Cossi M, Scalmani G, Rega N, Petersson GA, Nakatsuji H, Hada M, Ehara M, Toyota K, Fukuda R, Hasegawa J, Ishida M, Nakajima T, Honda Y, Kitao O, Nakai H, et al: Gaussian 03. Gaussian, Inc:: Wallingford, CT; 2004.

23. Lee C, Yang W, Parr RG: Development of the Colle-Salvetti correlationenergy formula into a functional of the electron density. Phys Rev 1988, B 37:785-789.

24. Becke AD: Density-functional exchange-energy approximation with correct asymptotic behavior. Phys Rev 1988, A 38:3098-3100.

25. Miehlich B, Savin A, Stoll H, Preuss H: Results obtained with the correlation energy density functionals of becke and Lee, Yang and Parr. Chem Phys Lett 1989, 157:200-206.

26. Krishnan R, Binkley JS, Seeger R, Pople JA: Self consistent molecular orbital methods. XX. A basis set for correlated wave functions. J Chem Phys $1980,72: 650-654$

27. Clark T, Chandrasekhar J, Spitznagel GW, Schleyer PvR: Efficient diffuse function-augmented basis sets for anion calculations. III. The $3-21+G$ basis set for first-row elements, Li-F. J Comput Chem 1983, 4:294-301.

28. Boys SF, Bernardi F: The calculation of small molecular interactions by the differences of separate total energies. Some procedures with reduced errors. Mol Phys 1970, 19:553-566.

29. Barone V, Cossi M, Tomasi J: Geometry optimization of molecular structures in solution by the polarizable continuum model. J Comput Chem 1998, 19:404-417.

30. Reed $A E$, Curtiss $L A$, Weinhold $F$ : Intermolecular interactions from a natural bond orbital, donor-acceptor viewpoint. Chem Rev 1988, 88:899-926.

31. Bullivant MJ, Gerald P: Photodecomposition of natural pyrethrins and related compounds. Pesticide Science 1976, 7:231-235.

doi:10.1186/1752-153X-5-66

Cite this article as: Elroby and Aziz: Understanding the decomposition reaction mechanism of chrysanthemic acid: a computational study. Chemistry Central Journal 2011 5:66. 